\title{
miR-320a upregulation contributes to the development of preeclampsia by inhibiting the growth and invasion of trophoblast cells by targeting interleukin 4
}

\author{
NING XIE ${ }^{1^{*}}$, ZHI JIA ${ }^{2^{*}}$ and $\mathrm{LI} \mathrm{LI}^{1}$ \\ Departments of ${ }^{1}$ Obstetrics and ${ }^{2}$ Anesthesiology, The Affiliated Hospital of \\ Jining Medical University, Jining, Shandong 272029, P.R. China
}

Received August 5, 2018; Accepted July 10, 2019

DOI: $10.3892 / \mathrm{mmr} .2019 .10574$

\begin{abstract}
Preeclampsia (PE) is a serious pregnancy-specific pathologic complication, and represents a primary cause of mother and fetus mortality. Abnormally expressed microRNAs (miRNAs) serve important regulatory roles in the development of PE. At present, the pathogenesis and molecular mechanism of PE remain unclear. The aim of the present study was to investigate the potential functions of miRNA (miR)-320a in the human extravillous trophoblast cell line HTR-8/SVneo and to identify the molecular mechanisms underlying miR-320a function. Reverse transcription-quantitative PCR was used in the present study to detect the levels of miR-320a in the placentas of 57 pregnant patients with PE and 57 healthy pregnant patients. The effects of miR-320a overexpression on the proliferation and invasion of HTR-8/SVneo cells were determined using MTT and Transwell invasion assays. Western blot analysis and dual luciferase reporter assay were used to identify the genes targeted by miR-320a. The present results suggested that miR-320a expression level was decreased in placentas of patients with PE and the expression level of miR-320a was found to be associated with the pathogenesis of $\mathrm{PE}(\mathrm{P}<0.05)$. Overexpression of miR-320a using miR-320a mimics significantly inhibited cell proliferation and invasion in HTR-8/SVneo cells in vitro $(\mathrm{P}<0.05)$. Furthermore, interleukin (IL)-4 was identified to be a direct target gene of miR-320a. miR-320a could repress IL-4 expression by binding to its 3 ' untranslated region $(\mathrm{P}<0.05)$. Mechanistic studies suggested that IL-4 was a functional target gene of miR-320a, and miR-320a upregulation inhibited the proliferation and
\end{abstract}

Correspondence to: Mrs. Li Li, Department of Obstetrics, The Affiliated Hospital of Jining Medical University, 89 Guhuai Road, Jining, Shandong 272029, P.R. China

E-mail: jyfylili@126.com

${ }^{*}$ Contributed equally

Key words: preeclampsia, microRNA-320a, development, HTR8/SVneo cells, interleukin-4 invasion of HTR-8/SVneo cells by directly targeting IL-4 $(\mathrm{P}<0.05)$. Collectively, to the best of our knowledge, the present study is the first to suggest that miR-320a may be a downregulated miRNA during PE, and IL-4 may act as a functional target gene of miR-320a. The present study suggested that miR-320a upregulation was involved in the development of PE by inhibiting the proliferation and invasion of trophoblast cells by targeting IL-4, indicating that the miR-320a/IL-4 pathway may represent a novel therapeutic target for PE treatment.

\section{Introduction}

Preeclampsia (PE) is a major hypertensive and clinical multisystem disorder during pregnancy, occurring in 3-5\% of pregnancies worldwide between 1980 and $2010(1,2)$. PE has become one of the leading causes of maternal and neonatal morbidity (2). Dysregulated differentiation of human trophoblast cells leads to abnormal cell invasion, causing PE (3-5). Major efforts have been made to identify the molecular mechanism underlying PE, and the development of PE is thought to be a multifactorial process, including placental and endothelial dysfunction, abnormal spiral artery remodeling, impaired trophoblast cells invasion and increased trophoblasts apoptosis (6). Dysfunction of human trophoblast cells has been suggested to be an important factor in PE pathogenesis, and understanding the potential mechanisms underlying trophoblast cell behavior may facilitate the identification of new therapeutic biomarker for PE.

MicroRNAs (miRNAs) are a class of endogenous and highly conserved small non-coding RNAs, which serve regulatory roles by inhibiting the expression of target genes by binding to specific regions of the 3 '-untranslated region (3'-UTR) of the target mRNAs, thus promoting transcript degradation and/or translational suppression $(7,8)$. Accumulating evidence suggested that miRNAs are associated with many important cellular events, including differentiation, growth, migration and apoptosis (9). Dysregulated miRNAs are found in tumor tissues and cell lines, and miRNAs have shown to act as tumor suppressor genes or oncogenes in various types of human cancer (10). Accumulating evidence has indicated that miRNAs serve an important role in the pathogenesis of PE (11). Xiao et al (12) demonstrated that miR-144 regulates 
the proliferation, invasion and migration of human trophoblast cells by targeting PTEN in PE. Wu et al (13) showed that miR-181a-5p suppresses the migration and invasion of human trophoblast cells in PE by directly targeting insulin like growth factor 2 mRNA binding protein 2. Dai et al (14) reported that miR-155 inhibits the proliferation and migration of human trophoblast cells by downregulating CCND1. In addition, a previous study found that miR-203 contributes to PE development in the human placenta by targeting vascular endothelial growth factor A, and miR-203 may be used as a potential therapeutic biomarker in treating PE (15). Although a large number of miRNAs have been previously described, the roles and molecular regulatory mechanisms of specific miRNAs in PE require further investigation.

A previous study demonstrated that the level of miR-320a is decreased in different tumor samples, and can function as a tumor suppressor by downregulating the expression level of multiple target genes (16). miR-320a acts as an important regulator in the development of gastric cancer by directly targeting Ras-related protein Rab-14 (17). miR-320a is downregulated in human non-small cell lung cancer and suppresses tumor cell proliferation and invasion by targeting insulin-like growth factor 1 receptor (18). miR-320a regulates high mobility group box 1 in hepatocellular carcinoma, limiting cell invasion and metastasis (19). In addition, miR-320a represses the progression of colorectal cancer by targeting Racl (20). However, to the best of our knowledge, no previous study investigated the biological functions and mechanisms of miR-320a in PE development.

The aim of the present study was to determine the potential roles and molecular mechanisms of miR-320a in the proliferation and invasion of the trophoblast cell line HTR-8/SVeno. The decreased levels of miR-320a were determined in placental samples collected from patients with $\mathrm{PE}$ by reverse transcription-quantitative PCR (RT-qPCR). MTT and invasion assays were performed to detected cell proliferation and invasion in HTR-8/SVneo cells after transfection with miR-320a mimic or mimic control. In addition, it was tested whether interleukin (IL)-4 was a direct target of miR-320a by dual luciferase report assay and western blots analysis. In addition, following rescue experiments, miR-320a upregulation was found to suppress proliferation and invasion of HTR-8/SVneo cells in PE at least partly by directly regulating IL-4.

\section{Materials and methods}

Patients. Placenta samples (gestational age, 32-40 weeks) were obtained from pregnant patients with $\mathrm{PE}(\mathrm{n}=57$, age 19-34 years) and healthy patients ( $\mathrm{n}=57$, age $22-31$ years) between January 2012 and December 2016 at the Department of Obstetrics, The Affiliated Hospital of Jining Medical University (Jining, China). The criteria for diagnosis of PE was as follows: Systolic blood pressure $\geq 140 \mathrm{mmHg}$ or diastolic blood pressure $\geq 90 \mathrm{mmHg}$ at 20 weeks post-gestation in a pregnant woman whose blood pressure was previously normal, $24 \mathrm{~h}$ urine protein $\geq 0.3 \mathrm{~g}$. Exclusion criteria were as follows: Hypertensive emergencies, blood system diseases and abnormal pregnancy with other complications of obstetrics. The clinical characteristics are presented in Table I. The placentas were immediately snap-frozen in liquid nitrogen after surgery and stored at $-80^{\circ} \mathrm{C}$ until further experimentation. The diagnosis of PE was based on The Royal
College of Obstetricians and Gynaecologists guidelines (1). The study was approved by the Ethics Committee of The Affiliated Hospital of Jining Medical University (2017TJ05749). Written consent was obtained from all patients.

Biochemical testing. Peripheral blood $(5 \mathrm{ml})$ was collected in EDTA anticoagulant tubes (Qiagen $\mathrm{GmbH}$ ) from all patients. Serum was acquired by centrifugation at 3,000 $\mathrm{x}$ g for $10 \mathrm{~min}$ at $37^{\circ} \mathrm{C}$. Body mass index was shown as weight divided by height squared $\left(\mathrm{kg} / \mathrm{m}^{2}\right)$. The $24 \mathrm{~h}$ urine protein level was quantified using a Fully Automated Chemistry Analyzer (AU400; Olympus Corporation).

Cell culture. The human extravillous trophoblast cells HTR-8/SVneo were purchased from the Cell Bank of The Chinese Academy of Sciences. HTR-8/SVneo cells were cultured in RPMI-1640 medium (Thermo Fisher Scientific, Inc.), and supplemented with $10 \%$ FBS (Thermo Fisher Scientific, Inc.), $100 \mu \mathrm{g} / \mathrm{ml}$ streptomycin and $100 \mathrm{U} / \mathrm{ml}$ penicillin in a humidified atmosphere with $5 \% \mathrm{CO}_{2}$ at $37^{\circ} \mathrm{C}$.

$R T-q P C R$. Total RNA was isolated from placenta samples and HTR-8/SVneo cells using TRIzol Reagent (Thermo Fisher Scientific, Inc.). Subsequently, RNA concentration was determined using a NanoDrop 2000 (Thermo Fisher Scientific, Inc.). RNA was reverse transcribed into cDNA using a PrimeScript RT Master Mix kit (Takara Bio, Inc.), according to the manufacturer's protocol. The thermocycling conditions were as follows: $95^{\circ} \mathrm{C}$ for $10 \mathrm{~min}, 42^{\circ} \mathrm{C}$ for $2 \mathrm{~min}, 37^{\circ} \mathrm{C}$ for $15 \mathrm{~min}$, $85^{\circ} \mathrm{C}$ for $5 \mathrm{sec}$ and $4^{\circ} \mathrm{C}$ for $30 \mathrm{~min}$. qPCR was performed using a SYBR PrimeScript RT-qPCR kit (Takara Bio, Inc.) on a Roche Light-cycler 480 real-time qPCR System (Roche Applied Science), according to the manufacturer's protocol. The thermocycling conditions of the qPCR were as follows: Initial denaturation at $95^{\circ} \mathrm{C}$ for $30 \mathrm{sec}$, followed by 40 cycles of $95^{\circ} \mathrm{C}$ for $10 \mathrm{sec}$ and $60^{\circ} \mathrm{C}$ for $30 \mathrm{sec}$. Primers for miR-320a, U6, IL-4 and GAPDH are presented in Table II. The primers were designed using Primer Premier software (version 5.0; Premier Biosoft International, Inc.), and purchased from Takara Biotechnology Co., Ltd. Expression level of miR-320a was normalized to the U6 expression level. IL-4 expression was normalized to GAPDH expression. Relative expression was calculated using the $2^{-\Delta \Delta \mathrm{Cq}}$ method (21).

Oligonucleotide and plasmid transfection. miR-320a mimic, mimic control, pcDNA3.1 empty vector and IL-4 overexpressing plasmid (pcDNA3.1-IL-4) were purchased from GenePharma Co., Ltd. The mimic control and pcDNA3.1 empty vector were used as negative controls for miR-320a mimic and pcDNA3.1-IL-4, respectively. The sequences of miR-320a mimic and mimic control were as follows: miR-320a mimic, 5'-AAAAGCUGGGUUGAGAGGGCGA-3'; mimic control, 5'-UUCUCCGAACGUGUCACGUTT-3'. For transfection, HTR-8/SVneo cells were inoculated in six-well plates at a density of $10^{5}$ cells/well, and were cultured in a humidified atmosphere with $5 \% \mathrm{CO}_{2}$ at $37^{\circ} \mathrm{C}$ overnight. Transient transfections of 100 pmol miR-320a mimic, 100 pmol mimic control, $2.5 \mu \mathrm{g}$ pcDNA3.1 empty vector and $2.5 \mu \mathrm{g}$ pcDNA3.1-IL-4 vector were performed using Lipofectamine 2000 (Invitrogen; Thermo Fisher Scientific, Inc.) at 70-80\% confluency according 
Table I. Clinical characteristics of patients with PE and healthy patients.

\begin{tabular}{lccc}
\hline Characteristics & PE pregnancies $(\mathrm{n}=57)$ & Normal pregnancies $(\mathrm{n}=57)$ & P-value \\
\hline Maternal age, years & $27.12 \pm 4.11$ & $26.37 \pm 3.29$ & 0.57 \\
$\mathrm{BMI}, \mathrm{kg} / \mathrm{m}^{2}$ & $24.91 \pm 2.78$ & $25.53 \pm 3.14$ & 0.61 \\
$\mathrm{SBP}, \mathrm{mmHg}$ & $154.06 \pm 7.65$ & $109.55 \pm 5.93$ & $<0.001$ \\
DBP, mmHg & $102.25 \pm 6.48$ & $70.69 \pm 5.07$ & $<0.001$ \\
24-h urine protein, g & $3.67 \pm 0.73$ & Not detected & $<0.001$ \\
Gestational age, weeks & $35.33 \pm 3.28$ & $39.25 \pm 2.16$ & 0.04 \\
Infant birth weight, $\mathrm{kg}$ & $2.27 \pm 0.50$ & $3.06 \pm 0.75$ & 0.02
\end{tabular}

PE, preeclampsia; BMI, body mass index; SBP, systolic blood pressure; DBP, diastolic blood pressure.

Table II. The sequences of primers for reverse transcriptionquantitative PCR assay.

\begin{tabular}{ll}
\hline Gene & \multicolumn{1}{c}{ Sequence $\left(5^{\prime}-3^{\prime}\right)$} \\
\hline miR-320a & F: GTTGGATCCGGCGTTTCCTTCCGACATG \\
& R: GCTGAATTCGTCCACTGCGGCTGTTCC \\
U6 & F: CTCGCTTCGGCAGCACA \\
& R: ACGCTTCACGAATTTGCGT \\
IL-4 & F: GCAGTTCCACAGGCACAA \\
& R: TGGTTGGCTTCCTTCACA \\
GAPDH & F: CAAGGTCATCCATGACAACT \\
& R: GTCCACCACCCTGTTGCTG
\end{tabular}

miR, microRNA; IL-4, Interleukin 4.

to the manufacturer's protocol. Cells were collected at $48 \mathrm{~h}$ after transfection for RT-qPCR analysis.

Target gene prediction. The potential target genes of miR-320a were identified using three publicly available algorithms, including TargetScanHuman version 7.2 (22), PicTar version 2007 (23) and miRanda version 2010 (24).

Dual luciferase reporter assay. Human IL-4 mRNA 3'-UTR, including the complementary binding sequence of miR-320a, (5'...UUAUGA GUUUUUGAUAGCUUUAU...-3'), was obtained from GenePharma Co., Ltd. and inserted into the pmirGLO vector (Promega Corporation) to construct the wild-type IL-4 reporter plasmid. The mutant IL-4 mRNA 3'-UTR (5'...UUAUGAGUUUUUGAUGAUCCCAU...-3') was also cloned into pmirGLO vector to construct the mutant IL-4 reporter plasmid. Co-transfection of the wild-type or the mutant IL-4 reporter plasmid and miR-320a mimic or mimic control was performed in HTR-8/SVneo cells using Lipofectamine 2000 (Invitrogen; Thermo Fisher Scientific, Inc.) according to the manufacturer's protocol. Renilla and Firefly luciferase values were detected at $24 \mathrm{~h}$ post-transfection using a dual luciferase reporter system kit (Promega Corporation) in a Varioskan Flash Microplate Reader (Thermo Fisher Scientific, Inc.), following the manufacturer's protocol. Renilla luciferase activity was used to normalize Firefly luciferase activity.

MTT assay. The proliferative ability of human trophoblast cells was assessed using an MTT assay. The transfected HTR-8/SVneo cells ( 8,000 cells/well) were inoculated into each well of 96-well plates. After $0,24,48$ and $72 \mathrm{~h}$ of transfection, $10 \mu \mathrm{l}$ MTT (5 mg/ml; Sigma-Aldrich; Merck KGaA) was added into each well. Then, the cells were incubated in a humidified atmosphere with $5 \% \mathrm{CO}_{2}$ at $37^{\circ} \mathrm{C}$ for $4 \mathrm{~h}$, and the supernatant was discarded. Subsequently, $150 \mu 1$ DMSO (Sigma-Aldrich; Merck KGaA) was added to dissolve the violet crystals for $10 \mathrm{~min}$ at $37^{\circ} \mathrm{C}$. A microplate reader (Bio-Rad Laboratories, Inc.) was used to detect absorbance of each well at $450 \mathrm{~nm}$ wavelength.

Transwell invasion assay. Transwell inserts (Costar; Corning, Inc.) precoated with Matrigel (BD Biosciences) were used to examine cell invasion. In total, $\sim 10^{5}$ transfected HTR-8/SVneo cells were inoculated in the upper well in $250 \mu$ l RPMI 1640 medium, whereas the lower well contained $500 \mu \mathrm{l}$ RPMI 1640 medium supplemented with 10\% FBS (Sigma-Aldrich; Merck KGaA). After a $24-\mathrm{h}$ incubation at $37^{\circ} \mathrm{C}$ with $5 \% \mathrm{CO}_{2}$, non-invasive cells attached to the upper side of membranes were removed using cotton swabs. The invasive cells were fixed with $100 \%$ methanol (Beyotime Institute of Biotechnology) for $20 \mathrm{~min}$ at $37^{\circ} \mathrm{C}$ and stained with $0.1 \%$ crystal violet (Beyotime Institute of Biotechnology) for $10 \mathrm{~min}$ at $37^{\circ} \mathrm{C}$. Stained cells were photographed and counted in five randomly-selected fields of view using a light microscope (DMI6000 B microscope; Leica Microsystems GmbH; magnification, x200).

Western blot analysis. Total protein was extracted from HTR-8/SVneo cells using RIPA buffer (Beyotime Institute of Biotechnology), and concentration of samples was detected using a bicinchoninic acid assay kit (Beyotime Institute of Biotechnology), according to the manufacturer's protocol. Subsequently, $10 \%$ SDS-PAGE was used to separate proteins $(30 \mu \mathrm{g})$ and proteins were transferred onto PVDF membranes (EMD Millipore). Then, membranes were treated with $5 \%$ non-fat milk for blocking for $60 \mathrm{~min}$ at $37^{\circ} \mathrm{C}$, and incubated overnight at $4^{\circ} \mathrm{C}$ with the following primary antibodies: Anti-IL-4 (cat. no. ab62351; 1:1,000; Abcam) and anti-GAPDH (cat. no. ab181602; 1:2,000; Abcam). Subsequently, membranes 

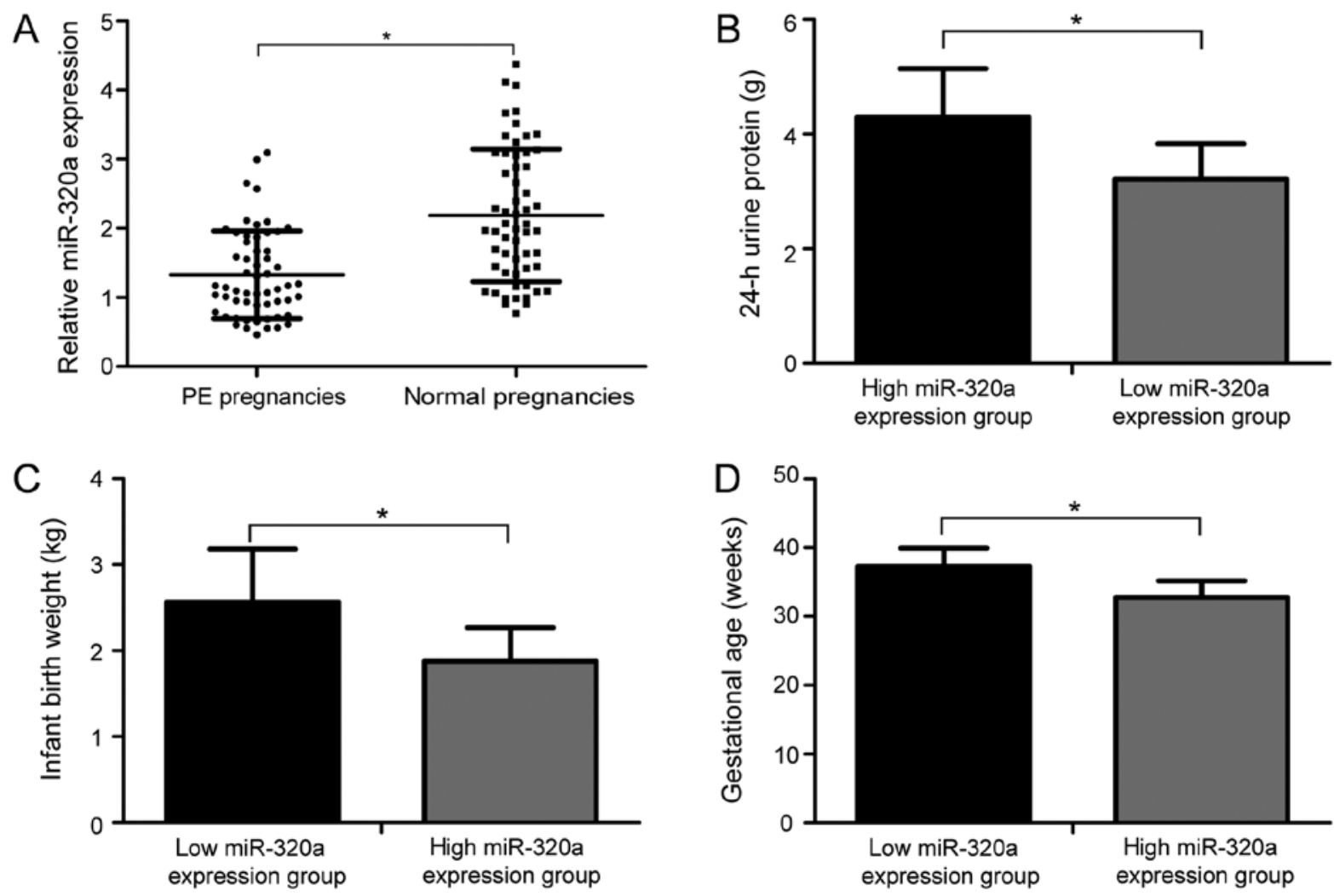

Figure 1. miR-320a is lowly expressed in placentas of patients with PE and is associated with the pathogenesis of PE. (A) miR-320a levels in the placentas of 57 patients with PE and 57 healthy patients were analyzed by reverse transcription-quantitative PCR assay. (B) Level of $24-\mathrm{h}$ urine protein in the high miR-320a expression group was higher than that in the low miR-320a expression group. (C) Infant birth weight in PE pregnancies with high miR-320a expression was lower than that in PE pregnancies with low miR-320a expression. (D) Gestational age was lower in the high miR-320a expression group compared with that in the low miR-320a expression group. ${ }^{\mathrm{P}}<0.05$. $\mathrm{PE}$, preeclampsia; miR, microRNA.

were incubated with horseradish peroxidase-conjugated goat anti-rabbit secondary antibody (cat. no. ab7090; 1:2,000; Abcam) at $37^{\circ} \mathrm{C}$ for $60 \mathrm{~min}$. Specific immunoreactive bands were visualized using a Pierce ECL western blotting kit (Thermo Fisher Scientific, Inc.). The positive bands were analyzed using ImageJ software (version 1.49; National Institutes of Health).

Statistical analysis. Statistical analysis was performed using SPSS 19.0 (IBM Corp.). Data are presented as the mean \pm SD. Each assay was conducted in triplicate. Student's t-test was performed to compare two groups. One-way ANOVA followed by Bonferroni's post hoc test was performed to compare multiple groups. $\mathrm{P}<0.05$ was considered to indicate a statistically significant difference.

\section{Results}

Clinical characteristics. Clinical characteristics were obtained from 57 pregnant patients with PE and 57 healthy patients. The clinical data are presented in Table I. The analysis of the clinical data suggested that diastolic blood pressure, systolic blood pressure and the level of $24 \mathrm{~h}$ urine protein in the PE group were higher than those in the healthy patients $(\mathrm{P}<0.05)$. The infant birth weight and gestational age were lower in the PE group than those in the healthy group $(\mathrm{P}<0.05)$. The maternal age and body mass index did not exhibit significant differences between the two groups. The high blood pressure and $24 \mathrm{~h}$ urine protein confirmed the diagnosis of PE.
miR-320a expression level is decreased in placentas of patients with PE. To investigate miR-320a expression level in placentas from patients with PE and healthy patients, RT-qPCR assay was performed. The relative levels of miR-320a were markedly downregulated in placentas from patients with PE compared with healthy patients (Fig. $1 \mathrm{~A} ; \mathrm{P}<0.05$ ). Additionally, patients with PE were divided into low and high miR-320a expression groups based on the mean $2^{-\Delta \Delta C q}$ value of miR-320a (1.32). Among the 57 patients with PE, 33 patients were in the low miR-320a expression group and 24 in the high miR-320a expression group. Interestingly, the analysis of the two groups suggested that the level of 24-h urine protein in the high miR-320a expression group was significantly higher than that in the low miR-320a expression group (Fig. 1B; $\mathrm{P}<0.05)$. The birth weight (Fig. 1C; $\mathrm{P}<0.05$ ) and gestational age (Fig. 1D; $\mathrm{P}<0.05)$ were lower in the high miR-320a expression group compared with the low expression group. The present data suggested that the expression level of miR-320a was decreased in placentas of patients with $\mathrm{PE}$ and was found to be associated with the pathogenesis of PE.

miR-320a overexpression represses the proliferation of HTR-8/SVneo cells. To identify the biological roles of miR-320a in PE, miR-320a mimic or mimic control were transfected into HTR-8/SVneo cells, and a MTT assay was performed to examine the effects of miR-320a on the proliferation of HTR-8/SVneo cells in vitro. RT-qPCR analysis showed that miR-320a expression levels in HTR-8/SVneo treated with 
A

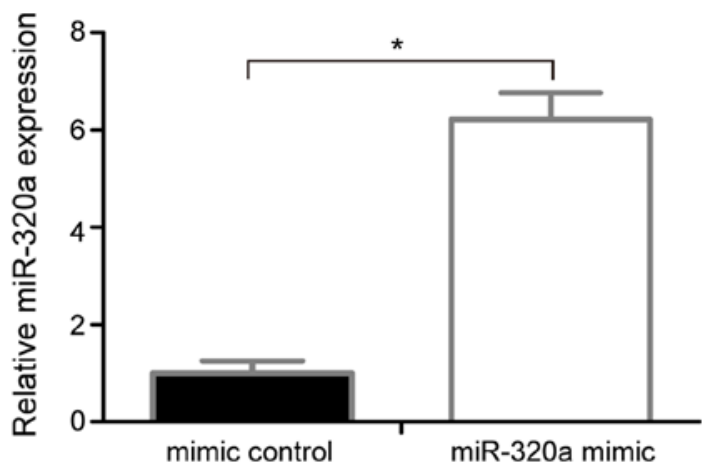

C

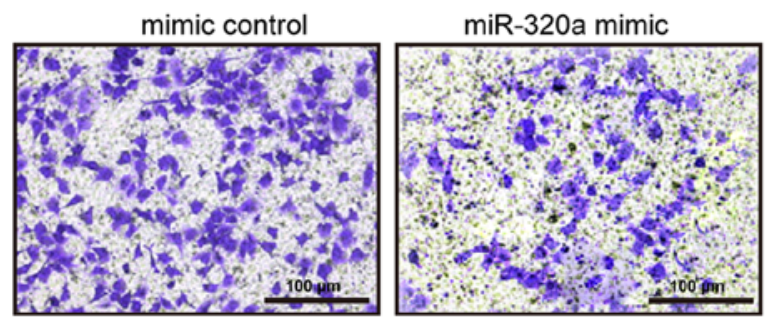

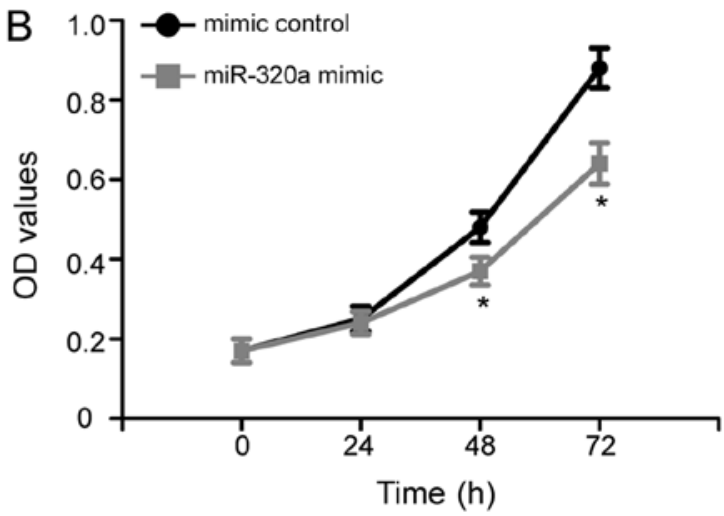

D

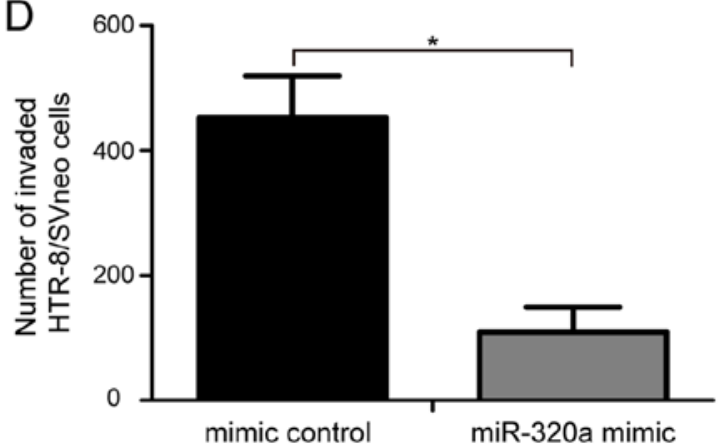

Figure 2. miR-320a overexpression represses the proliferation and invasion of HTR-8/SVneo cells. (A) Expression levels of miR-320a were detected in HTR-8/SVneo cells after transfection with miR-320a mimic or mimic control. (B) Cell proliferation was examined by MTT assay in HTR-8/SVneo cells transfected with miR-320a mimic or mimic control. (C) Invasive ability in HTR-8/SVneo cells was detected using a Transwell invasion assay following transfection with miR-320a mimic or mimic control. Magnification, x200. Scale bar, $100 \mu \mathrm{m}$. (D) Overexpression of miR-320a inhibited the invasive ability of HTR-8/SVneo cells compared with the mimic control. ${ }^{*} \mathrm{P}<0.05$ vs. mimic control. miR, microRNA; OD, optical density.

miR-320a mimic were significantly upregulated compared with cells transfected with control mimic (Fig. 2A; $\mathrm{P}<0.05$ ). Compared with cells transfected with control mimic, cells transfected with miR-320a exhibited a significantly decrease in cell proliferation at 48 and $72 \mathrm{~h}$ (Fig. $2 \mathrm{~B}$; $\mathrm{P}<0.05$ ). The present data suggested that the upregulation of miR-320a is involved in the development of PE by inhibiting the proliferation of HTR-8/SVneo cells.

miR-320a overexpression reduces the invasionofHTR-8/SVneo cells. To identify the possible function of miR-320a on human trophoblasts invasion, HTR-8/SVneo cells were transfected with either miR-320a mimic or mimic control and a Transwell invasion assay was performed (Fig. 2C). The present data suggested that over-expression of miR-320a markedly inhibited the invasive ability of HTR-8/SVneo cells compared with the mimic control (Fig. 2D; $\mathrm{P}<0.05$ ). The present results demonstrated that the upregulation of miR-320a is involved in the development of PE by inhibiting the invasion of HTR-8/SVneo cells.

IL-4 is a direct target gene of miR-320a in HTR-8/SVneo cells. To investigate the molecular mechanism involved in the miR-320a-mediated regulation of proliferation and invasion of human trophoblasts, three computational algorithms, including TargetScanHuman, miRanda and PicTar, were used to analyze the possible targets of miR-320a. A previous study showed that IL-4 polymorphisms are associated with PE (25), and IL-4 was found to have a high score in the three computational algorithms. Therefore, IL-4 was selected as a candidate target gene of miR-320a for further analysis. To evaluate whether IL-4 was directly regulated by miR-320a, its 3'-UTR containing the complementary binding sequence of miR-320a, and a mutant 3'-UTR containing mutations in the miR-320a binding sequence, were introduced into the pmirGLO vector (Fig. 3A). After co-transfection with the wild-type IL-4 reporter plasmid and miR-320a mimic into HTR-8/SVneo cells, the luciferase activity ratio was significantly decreased, whereas co-transfection with mutant IL-4 reporter plasmid and miR-320a mimic did not affect the luciferase activity of HTR-8/SVneo cells (Fig. 3B). Additionally, the effect of miR-320a on IL-4 expression was also determined using western blot analysis, and the present results suggested that miR-320a mimic transfection significantly suppressed the protein expression level of IL-4 in HTR-8/SVneo cells (Fig. 3C; $\mathrm{P}<0.05$ ). The present results indicated that IL-4 was a direct target gene of miR-320a in HTR-8/SVneo cells.

miR-320a inhibits the proliferation and invasion of HTR-8/SVneo cells by directly targeting $I L-4$. To examine whether the inhibitory effects of miR-320a on cell proliferation and invasion of human trophoblasts were mediated by IL-4 downregulation, IL-4 was overexpressed by co-transfecting pcDNA3.1-IL-4 vector with miR-320a mimic in HTR-8/SVneo cells. Western blot analysis suggested that transfection of pcDNA3.1-IL-4 vector significantly increased the expression level of IL-4 in HTR-8/SVneo cells (Fig. 4A; $\mathrm{P}<0.05)$. In addition, the protein expression level of IL-4 in 
A

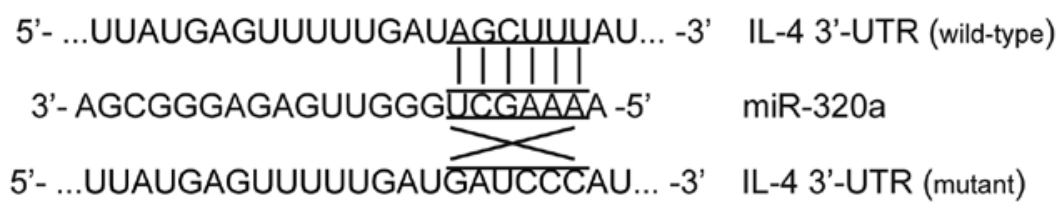

B
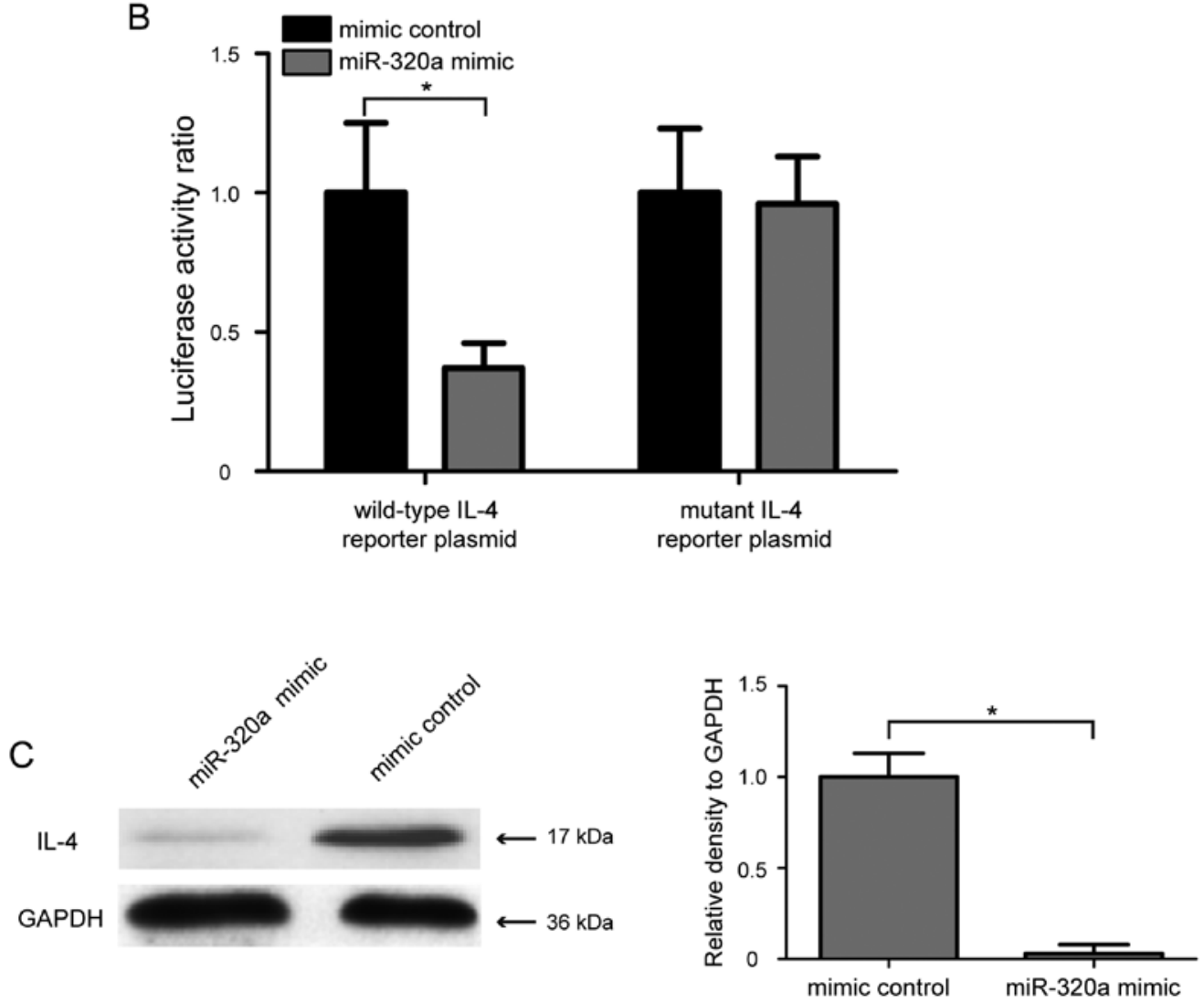

Figure 3. IL-4 is a direct target of miR-320a in HTR-8/SVneo cells. (A) Wild-type and mutant 3'-UTR sequences of IL-4 mRNA interacting with miR-320a (B) Luciferase activity ratio was determined using a dual luciferase reporter assay in HTR-8/SVneo cells co-transfected with wild-type or mutant IL-4 reporter plasmid and miR-320a mimic or mimic control. (C) Relative protein expression level of IL-4 in HTR-8/SVneo cells transfected with miR-320a mimic or mimic control was detected using western blotting. miR-320a mimic transfection suppressed the protein level of IL-4 in HTR-8/SVneo cells. " $\mathrm{P}<0.05$. miR, microRNA; IL, interleukin; UTR, untranslated region.

HTR-8/SVneo cells was also increased following co-transfection of the pcDNA3.1-IL-4 vector with miR-320a mimic (Fig. 4B; P<0.05). Notably, restoring the expression level of IL-4 partially decreased the inhibitory effect of miR-320a overexpression on HTR-8/SVneo cell proliferation (Fig. 4C; $\mathrm{P}<0.05)$. Consistent with the cell proliferation results, overexpression of IL-4 in HTR-8/SVneo cells reduced the inhibitory effect of miR-320a overexpression on cell invasion (Fig. 4D; $\mathrm{P}<0.05$ ). Collectively, the present data suggested that IL-4 was a functional target gene of miR-320a, and miR-320a upregulation inhibited the proliferation and invasion of HTR-8/SVneo cells by directly targeting IL- 4 .

\section{Discussion}

The molecular mechanisms of PE pathogenesis are complex and are thought to be multifactorial processes (3-5). Abnormal proliferation and invasion of human trophoblasts serve crucial roles in the development of PE (26). Therefore, it is important to study the mechanism of trophoblast proliferation and invasion in order to identify novel reliable and effective molecular targets for treating PE. Accumulating evidence has indicated that miRNAs are dysregulated in placentas of patients with PE $(27,28)$. The identification of dysregulated miRNAs and their target genes in the placenta of patients with PE has provided a basis for investigating the mechanisms underlying PE development $(29,30)$. To the best of our knowledge, the present study was the first to suggest that miR-320a may be downregulated in patients with PE, and IL-4 was identified as a functional target gene of miR-320a. The present study suggested that miR-320a upregulation was involved in the development of PE by inhibiting the proliferation and invasion of trophoblast cells by targeting IL-4.

miR-320a has been found to be dysregulated in cerebral ischemia, myasthenia gravis and human tumors (31). Recently, several reports have demonstrated that miR-320a acts as a tumor suppressor gene by targeting Neuropilin 1 in colorectal cancer (32), Myc in hepatocellular carcinoma (33), 

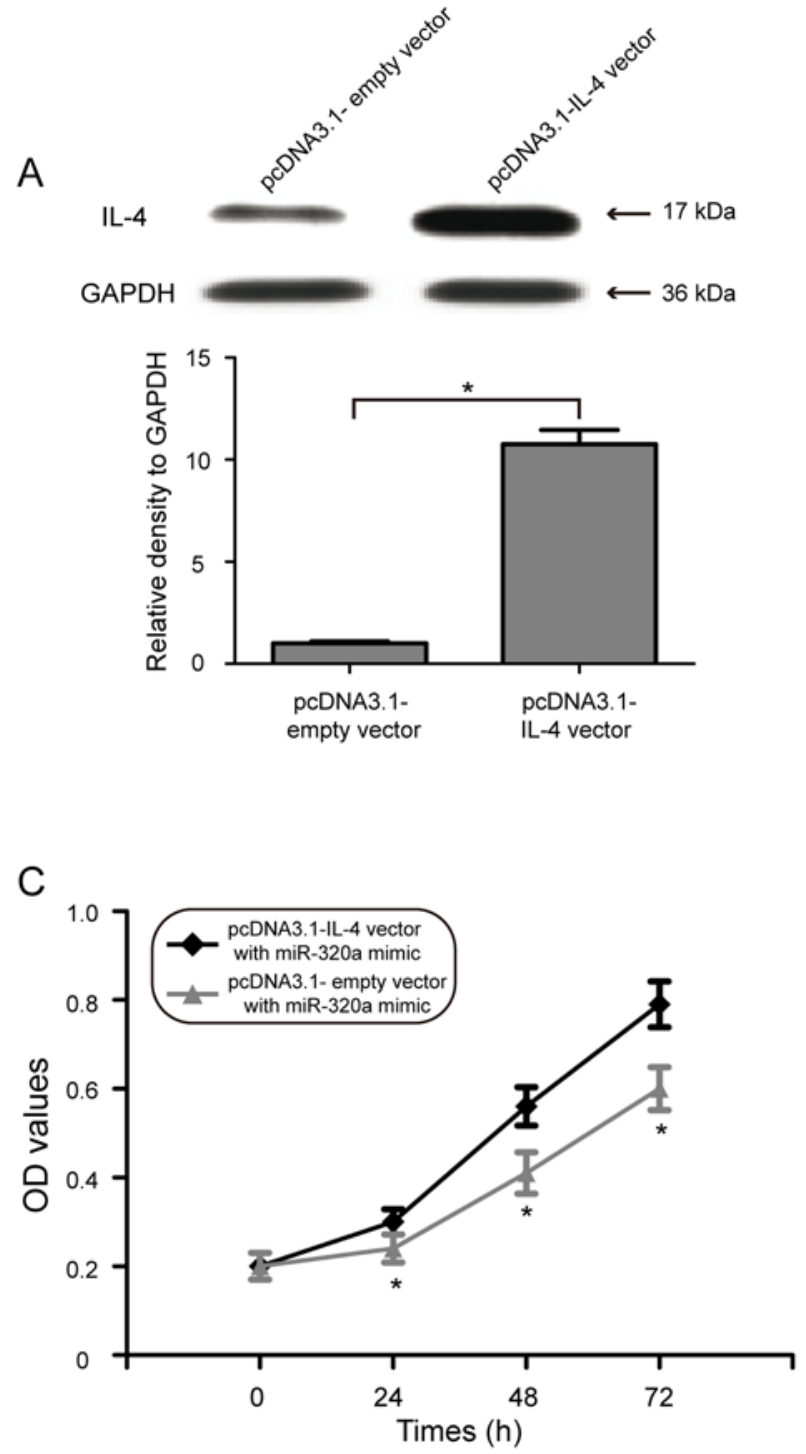
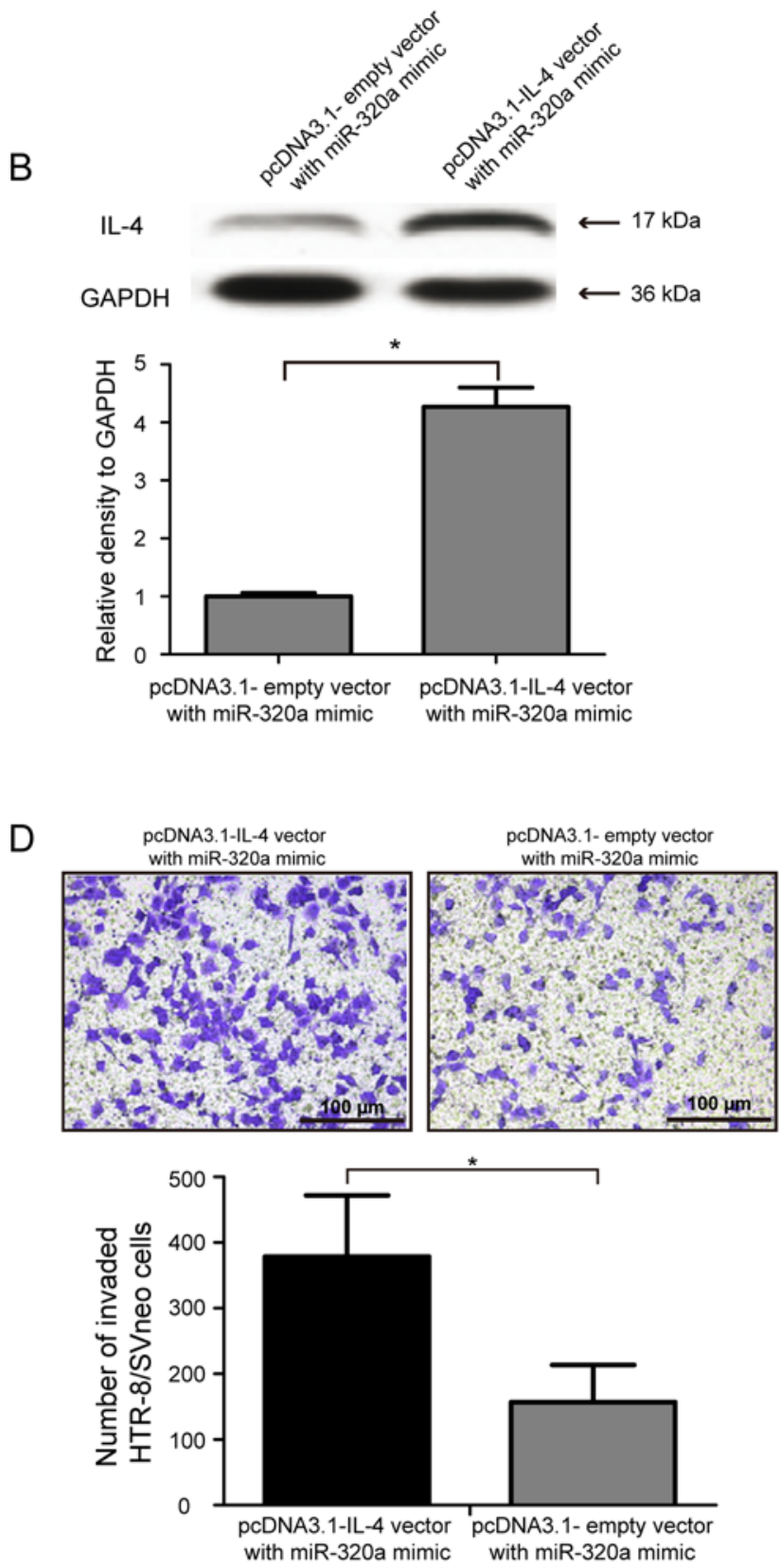

Figure 4. miR-320a inhibits the proliferation and invasion of HTR-8/SVneo cells by directly targeting $I L-4$. (A) Western blot analysis of IL-4 expression in HTR-8/SVneo cells following transfection with pcDNA3.1-IL-4 vector or pcDNA3.1- empty vector. (B) Protein levels of IL-4 in HTR-8/SVneo cells were significantly increased following transfection with pcDNA3.1-IL-4 vector and miR-320a mimic compared with pcDNA3.1-empty vector with miR-320a mimic group. ${ }^{*} \mathrm{P}<0.05$. (C) Restoring IL-4 expression partially reduced the suppressive effect of miR-320a overexpression on HTR-8/SVneo cell proliferation. ${ }^{*} \mathrm{P}<0.05$ vs. pcDNA3.1-IL-4 vector with miR-320a mimic. (D) Invasive ability of HTR-8/SVneo cells co-transfected with pcDNA3.1-IL-4 vector or pcDNA3.1-empty vector with miR-320a mimic was determined using a Transwell invasion assay. " $\mathrm{P}<0.05$. miR, microRNA; IL, interleukin; UTR, untranslated region; OD, optical density.

and staphylococcal nuclease and tudor domain containing 1 in gliomas (34). Since miR-320a regulates multiple target genes, it may serve an important role in the regulatory network underlying the development of PE. To the best of our knowledge, the functions and potential mechanisms of miR-320a in PE have not been previously identified. The present results were in line with previous studies (32-34) suggesting that miR-320a is a downregulated miRNA in patients with PE. The present statistical analysis showed that patients with PE with high miR-320a expression had a higher level of 24-h urine protein, their children presented a lower birth weight, and the gestational age was decreased compared with patients with PE in the low miR-320a expression group, indicating that miR-320a may be associated with the pathogenesis of PE.

Furthermore, the function of miR-320a on cell proliferation and invasion were analyzed by using MTT and Transwell invasion assays, respectively, in HTR-8/SVneo cells after transfection with miR-320a mimic and mimic control. The present results suggested that transient overexpression of miR-320a induced a significant decrease in HTR-8/SVneo cell proliferation and invasion, suggesting that the upregulation of miR-320a is involved in the development of PE by inhibiting the proliferation and invasion of HTR-8/SVneo 
cells. The present results provided novel insights into the roles of miR-320a in PE development. miRNAs act by silencing their target genes; therefore, the identification of the potential targets of miR-320a was required in order to elucidate the mechanisms underlying miR-320a-mediated PE development. Using three bioinformatic tools, the potential target genes of miR-320a have been analyzed. IL-4 was selected for further experiments as it ranked the highest amongst the potential target genes. In addition, the association between IL-4 polymorphisms and PE was previously investigated (25). T helper 2 (Th2) cells produce IL-13, IL-6, IL-5 and IL-4, and these cytokines are associated with the production of antibody and inhibition of cell-mediated immunity (35). A previous study suggested that PE is associated with an overproduction of Th2-specific cytokines (36). An increased expression level of IL-4 is involved in PE during the second half of the pregnancy, probably by decreasing nitric oxide secretion and promoting human leukocyte antigen expression $(37,38)$. To investigate this possibility, wild-type or mutant IL-4 reporter plasmid and miR-320a mimic or mimic control were transfected into HTR-8/SVneo cells, and Renilla and Firefly luciferase values were detected using a dual luciferase reporter assay. The present results suggested that miR-320a could repress IL-4 expression by binding to its 3'-UTR. Moreover, western blot assay suggested that IL-4 protein expression was significantly reduced in HTR-8/SVneo cells following transfection with miR-320a mimic. The present results suggested that IL-4 was a direct target gene of miR-320a in HTR-8/SVneo cells.

In addition, rescue experiments were performed to investigate whether miR-320a exerted its function via IL-4 downregulation in HTR-8/SVneo cells. The present results suggested that overexpression of IL-4 partly reversed the inhibitory effects of miR-320a on the proliferation and invasion of human trophoblasts. Collectively, the present data suggested that miR-320a was involved in the development of preeclampsia by targeting IL-4, suggesting that the miR-320a/IL-4 pathway may be a novel therapeutic target for PE treatment. Notably, the target genes of miR-320a are multiple and are not limited to IL-4; therefore, other potential target genes need to be investigated to further examine the regulatory network of miR-320a in the development of PE. The main limitation of the present study is that the molecular mechanisms underlying IL-4-regulated cell proliferation and invasion in human trophoblasts were not investigated and require further investigation.

Collectively, the present study suggested that miR-320a was lowly expressed in the placentas of patients with PE and miR-320a may be involved in the development of PE by inhibiting the proliferation and invasion of trophoblast cells by targeting IL-4. Therefore, therapeutic approaches targeting the miR-320a/IL-4 pathway may improve the treatment of PE.

\section{Acknowledgements}

Not applicable.

\section{Funding}

No funding was received.

\section{Availability of data and materials}

The datasets used and/or analyzed during the current study are available from the corresponding author on reasonable request.

\section{Authors' contributions}

NX and LL designed and carried out the experiments. NX and ZJ analyzed the data. LL and ZJ wrote and revised the manuscript. All authors read and approved the final manuscript.

\section{Ethics approval and consent to participate}

The present study was approved by The Affiliated Hospital of Jining Medical University (Jining, China). Written consent was obtained from all patients.

\section{Patient consent for publication}

Written informed consent was obtained from all patients prior to the start of the study.

\section{Competing interests}

The authors declare that they have no competing interest.

\section{References}

1. Redman CW and Sargent IL: Latest advances in understanding preeclampsia. Science 308: 1592-1594, 2005.

2. Mol BWJ, Roberts CT, Thangaratinam S, Magee LA, de Groot CJM and Hofmeyr GJ: Pre-eclampsia. Lancet 387: 999-1011, 2016.

3. AmaralLM,WallaceK,Owens MandLaMarca B:Pathophysiology and current clinical management of preeclampsia. Curr Hypertens Rep 19: 61, 2017.

4. Ji L, Brkic J, Liu M, Fu G, Peng C and Wang YL: Placental trophoblast cell differentiation: Physiological regulation and pathological relevance to preeclampsia. Mol Aspects Med 34: 981-1023, 2013.

5. Staun-Ram E and Shalev E: Human trophoblast function during the implantation process. Reprod Biol Endocrinol 3: 56, 2005.

6. Noris M, Perico N and Remuzzi G: Mechanisms of disease: Pre-eclampsia. Nat Clin Pract Nephrol 1: 98-114; quiz 120, 2005.

7. He L and Hannon GJ: MicroRNAs: Small RNAs with a big role in gene regulation. Nat Rev Genet 5: 522-531, 2004.

8. Bartel DP: MicroRNAs: Genomics, biogenesis, mechanism, and function. Cell 116: 281-297, 2004.

9. Schickel R, Boyerinas B, Park SM and Peter ME: MicroRNAs: Key players in the immune system, differentiation, tumorigenesis and cell death. Oncogene 27: 5959-5974, 2008.

10. Shenouda SK and Alahari SK: MicroRNA function in cancer: Oncogene or a tumor suppressor? Cancer Metastasis Rev 28: 369-378, 2009.

11. Chen DB and Wang W: Human placental microRNAs and preeclampsia. Biol Reprod 88: 130, 2013.

12. Xiao J, Tao T, Yin Y, Zhao L, Yang L and Hu L: miR-144 may regulate the proliferation, migration and invasion of trophoblastic cells through targeting PTEN in preeclampsia. Biomed Pharmacother 94: 341-353, 2017.

13. Wu L, Song WY, Xie Y, Hu LL, Hou XM, Wang R, Gao Y, Zhang JN, Zhang L, Li WW, et al: miR-181a-5p suppresses invasion and migration of HTR-8/SVneo cells by directly targeting IGF2BP2. Cell Death Dis 9: 16, 2018.

14. Dai Y, Qiu Z, Diao Z, Shen L, Xue P, Sun H and Hu Y: MicroRNA-155 inhibits proliferation and migration of human extravillous trophoblast derived HTR-8/SVneo cells via down-regulating cyclin D1. Placenta 33: 824-829, 2012.

15. Liu F, Wu K, Wu W, Chen Y, Wu H, Wang H and Zhang W: miR203 contributes to preeclampsia via inhibition of VEGFA expression. Mol Med Rep 17: 5627-5634, 2018. 
16. Sun L, Liu B, Lin Z, Yao Y, Chen Y, Li Y, Chen J, Yu D, Tang Z, Wang B, et al: miR-320a acts as a prognostic factor and inhibits metastasis of salivary adenoid cystic carcinoma by targeting ITGB3. Mol Cancer 14: 96, 2015.

17. Li Y, Liu H, Shao J and Xing G: miR-320a serves as a negative regulator in the progression of gastric cancer by targeting RAB14. Mol Med Rep 16: 2652-2658, 2017.

18. Wang J, Shi C, Cao L, Zhong L and Wang D: MicroRNA-320a is downregulated in non-small cell lung cancer and suppresses tumor cell growth and invasion by directly targeting insulin-like growth factor 1 receptor. Oncol Lett 13: 3247-3252, 2017.

19. Lv G, Wu M, Wang M, Jiang X, Du J, Zhang K, Li D, Ma N, Peng Y, Wang L, et al: miR-320a regulates high mobility group box 1 expression and inhibits invasion and metastasis in hepatocellular carcinoma. Liver Int 37: 1354-1364, 2017.

20. Zhao H, Dong T, Zhou H, Wang L, Huang A, Feng B, Quan Y, Jin R, Zhang W, Sun J, et al: miR-320a suppresses colorectal cancer progression by targeting Rac1. Carcinogenesis 35 886-895, 2014.

21. Livak KJ and Schmittgen TD: Analysis of relative gene expression data using real-time quantitative PCR and the 2(-Delta Delta C(T)) method. Methods 25: 402-408, 2001.

22. Agarwal V, Bell GW, Nam JW and Bartel DP: Predicting effective microRNA target sites in mammalian mRNAs. Elife 4, 2015.

23. Krek A, Grun D, Poy MN, Wolf R, Rosenberg L, Epstein EJ, MacMenamin P, da Piedade I, Gunsalus KC, Stoffel $M$ and Rajewsky N: Combinatorial microRNA target predictions. Nat Genet 37: 495-500, 2005.

24. John B, Enright AJ, Aravin A, Tuschl T, Sander C and Marks DS: Human MicroRNA targets. PLoS Biol 2: e363, 2004.

25. Chen J, Zhong M and Yu YH: Association between interleukin-4 polymorphisms and risk of pre-eclampsia in a population of Chinese pregnant women. Genet Mol Res 16, 2017.

26. Zhang Z, Wang $X$, Zhang L, Shi Y, Wang $J$ and Yan $H$ : $\mathrm{Wnt} / \beta$-catenin signaling pathway in trophoblasts and abnormal activation in preeclampsia (Review). Mol Med Rep 16: 1007-1013, 2017.

27. Pineles BL, Romero R, Montenegro D, Tarca AL, Han YM, Kim YM, Draghici S, Espinoza J, Kusanovic JP, Mittal P, et al: Distinct subsets of microRNAs are expressed differentially in the human placentas of patients with preeclampsia. Am J Obstet Gynecol 196: 261, e1-e6, 2007.
28. Zhu XM, Han T, Sargent IL, Yin GW and Yao YQ: Differential expression profile of microRNAs in human placentas from preeclamptic pregnancies vs. normal pregnancies. Am J Obstet Gynecol 200: 661, e1-e7, 2009.

29. Jairajpuri DS and Almawi WY: MicroRNA expression pattern in pre-eclampsia (Review). Mol Med Rep 13: 2351-2358, 2016.

30. Noguer-Dance M, Abu-Amero S, Al-Khtib M, Lefèvre A, Coullin P, Moore GE and Cavaillé J: The primate-specific microRNA gene cluster (C19MC) is imprinted in the placenta. Hum Mol Genet 19: 3566-3582, 2010.

31. Lu C, Liao Z, Cai M and Zhang G: MicroRNA-320a downregulation mediates human liver cancer cell proliferation through the Wnt/beta-catenin signaling pathway. Oncol Lett 13: 573-578, 2017.

32. Zhang Y, He X, Liu Y, Ye Y, Zhang H, He P, Zhang Q, Dong L, Liu Y and Dong J: microRNA-320a inhibits tumor invasion by targeting neuropilin 1 and is associated with liver metastasis in colorectal cancer. Oncol Rep 27: 685-694, 2012.

33. Xie F, Yuan Y, Xie L, Ran P, Xiang X, Huang Q, Qi G, Guo X, Xiao $\mathrm{C}$ and Zheng S: miRNA-320a inhibits tumor proliferation and invasion by targeting c-Myc in human hepatocellular carcinoma. Onco Targets Ther 10: 885-894, 2017.

34. Li H, Yu L, Liu J, Bian X, Shi C, Sun C, Zhou X, Wen Y, Hua D, Zhao S, et al: miR-320a functions as a suppressor for gliomas by targeting SND1 and beta-catenin, and predicts the prognosis of patients. Oncotarget 8: 19723-19737, 2017.

35. Singh VK and Rai G: Cytokines in posterior uveitis: An update. Immunol Res 23: 59-74, 2001.

36. Kang L, Chen $\mathrm{CH}$, Yu CH, Chang $\mathrm{CH}$ and Chang FM: An association study of interleukin-4 gene and preeclampsia in Taiwan. Taiwan J Obstet Gynecol 53: 215-219, 2014.

37. Hashemi V, Dolati S, Hosseini A, Gharibi T, Danaii S and Yousefi M: Natural killer T cells in Preeclampsia: An updated review. Biomed Pharmacother 95: 412-418, 2017.

38. Vianna P, Mondadori AG, Bauer ME, Dornfeld D and Chies JA: HLA-G and $\mathrm{CD} 8^{+}$regulatory $\mathrm{T}$ cells in the inflammatory environment of pre-eclampsia. Reproduction 152: 741-751, 2016.

This work is licensed under a Creative Commons Attribution-NonCommercial-NoDerivatives 4.0 International (CC BY-NC-ND 4.0) License. 